\title{
Combining Discrete and Neural Features for Sequence Labeling
}

\author{
Jie Yang, Zhiyang Teng, Meishan Zhang, and Yue Zhang^ \\ Singapore University of Technology and Design \\ \{jie_yang, zhiyang_teng\}@mymail.sutd.edu.sg \\ \{meishan_zhang, yue_zhang\}@sutd.edu.sg
}

\begin{abstract}
Neural network models have recently ${ }^{1}$ received heated research attention in the natural language processing community. Compared with traditional models with discrete features, neural models have two main advantages. First, they take low-dimensional, real-valued embedding vectors as inputs, which can be trained over large raw data, thereby addressing the issue of feature sparsity in discrete models. Second, deep neural networks can be used to automatically combine input features, and including non-local features that capture semantic patterns that cannot be expressed using discrete indicator features. As a result, neural network models have achieved competitive accuracies compared with the best discrete models for a range of NLP tasks.

On the other hand, manual feature templates have been carefully investigated for most NLP tasks over decades and typically cover the most useful indicator pattern for solving the problems. Such information can be complementary the features automatically induced from neural networks, and therefore combining discrete and neural features can potentially lead to better accuracy compared with models that leverage discrete or neural features only.

In this paper, we systematically investigate the effect of discrete and neural feature combination for a range of fundamental NLP tasks based on sequence labeling, including word segmentation, POS tagging and named entity recognition for Chinese and English, respectively. Our results on standard benchmarks show that state-of-the-art neural models can give accuracies comparable to the best discrete models in the literature for most tasks and combing discrete and neural features unanimously yield better results.
\end{abstract}

Keywords: Discrete Features, Neural Features, LSTM

\section{Introduction}

There has been a surge of interest in neural methods for natural language processing over the past few years. Neural models have been explored for a wide

\footnotetext{
* Corresponding author

${ }^{1}$ This paper was accepted by International Conference on Intelligent Text Processing and Computational Linguistics (CICLing) 2016, April; Konya, Turkey.
} 
range of tasks, including parsing [1|2|3|4|5|6|7, machine translation [8|9|10|11|12]13, sentiment analysis [14|15/16 17/18] and information extraction [19/20|21/22], achieving results competitive to the best discrete models.

Compared with discrete models with manual indicator features, the main advantage of neural networks is two-fold. First, neural network models take low-dimensional dense embeddings 23|24|25 as inputs, which can be trained from large-scale test, thereby overcoming the issue of sparsity. Second, non-linear neural layers can be used for combining features automatically, which saves the expense of feature engineering. The resulting neural features can capture complex non-local syntactic and semantic information, which discrete indicator features can hardly encode.

On the other hand, discrete manual features have been studied over decades for many NLP tasks, and effective feature templates have been well-established for them. This source of information can be complementary to automatic neural features, and therefore a combination of the two feature sources can led to improved accuracies. In fact, some previous work has attempted on the combination. Turian et al. 26] integrated word embedding as real-word features into a discrete Conditional Random Field [27] (CRF) model, finding enhanced results for a number of sequence labeling tasks. Guo et al. 28, show that the integration can be improved if the embedding features are carefully discretized. On the reverse direction, Ma et al. 29] treated a discrete perception model as a neural layer, which is integrated into a neural model. Wang \& Manning [30] integrated a discrete CRF model and a neural CRF model by combining their output layers. Greg \& Dan [6] and Zhang et al. [18] also followed this method. Zhang \& Zhang [31] compared various integration methods for parsing, and found that the second type of integration gives better results.

We follow Zhang \& Zhang [31, investigating the effect of feature combination for a range of sequence labeling tasks, including word segmentation, Part-OfSpeech (POS) tagging and named entity recognition (NER) for Chinese and English, respectively. For discrete features, we adopt a CRF model with stateof-the art features for each specific task. For neural features, we adopt a neural CRF model, using a separated Long Short-Term Memory [32] (LSTM) layer to extract input features. We take standard benchmark datasets for each task. For all the tasks, both the discrete model and the neural model give accuracies that are comparable to the state-of-the-art. A combination of discrete and neural feature, gives significantly improved results with no exception.

The main contributions that we make in this investigation include:

- We systematically investigate the effect of discrete and neural feature combination for a range of fundamental NLP tasks, showing that the two types of feature are complimentary.

- We systematically report results of a state-of-the-art neural network sequence labeling model on the NLP tasks, which can be useful as reference to future work.

- We report the best results in the literatures for a number of classic NLP tasks by exploiting neural feature integration. 
- The source code of the LSTM and CRF implementations of this paper are released under GPL at https://github.com/SUTDNLP/NNSegmentation, ../NNPOSTagging and ../NNNamedEntity .

\section{Related Work}

There has been two main kinds of methods for word segmentation. Xue 33 . treat it as a sequence labeling task, using $\mathrm{B}($ egin)/I(nternal) /E(nding)/S(inglecharacter word) tags on each character in the input to indicate its segmentation status. The method was followed by Peng et al. [34, who use CRF to improve the accuracies. Most subsequent work follows [34/35/36/37/38 and feature engineering has been out of the key research questions. This kind of research is commonly referred to as the character-based method. Recently, neural networks have been applied to character-based segmentation [39]40|41], giving results comparable to discrete methods. On the other hand, the second kind of work studies wordbased segmentation, scoring outputs based on word features directly [42 43/44]. We focus on the character-based method, which is a typical sequence labeling problem.

POS-tagging has been investigated as a classic sequence labeling problem 45 46 47, for which a well-established set of features are used. These handcrafted features basically include words, the context of words, word morphologies and word shapes. Various neural network models have also been used for this task. In order to include word morphology and word shape knowledge, a convolutional neural network $(\mathrm{CNN})$ for automatically learning character-level representations is investigated in [48]. Collobert et al. [25] built a CNN neural network for multiple sequence labeling tasks, which gives state-of-the-art POS results. Recurrent neural network models have also been used for this task 49:50. Huang et al. [50] combines bidirectional LSTM with a CRF layer, their model is robust and has less dependence on word embedding.

Named entity recognition is also a classical sequence labeling task in the NLP community. Similar to other tasks, most works access NER problem through feature engineering. McCallum \& Li [51] use CRF model for NER task and exploit Web lexicon as feature enhancement. Chieu \& Ng [52, Krishnan \& Manning 53] and Che et al. 54 tackle this task through non-local features [55]. Besides, many neural models, which are free from handcrafted features, have been proposed in recent years. In Collobert et al. 25] model we referred before, NER task has also been included. Santos et al. [56] boost the neural model by adding character embedding on Collobert's structure. James et al. [57] take the lead by employing LSTM for NER tasks. Chiu et al. [58] use CNN model to extract character embedding and attach it with word embedding and afterwards feed them into Bi-directional LSTM model. Through adding lexicon features, Chiu's NER system get state-of-the-art performance on both CoNLL2003 and OntoNotes 5.0 NER datasets. 


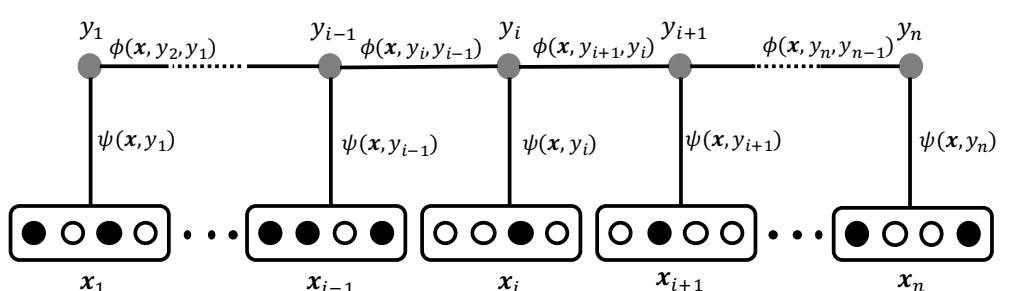

(a) Discrete Model Structure

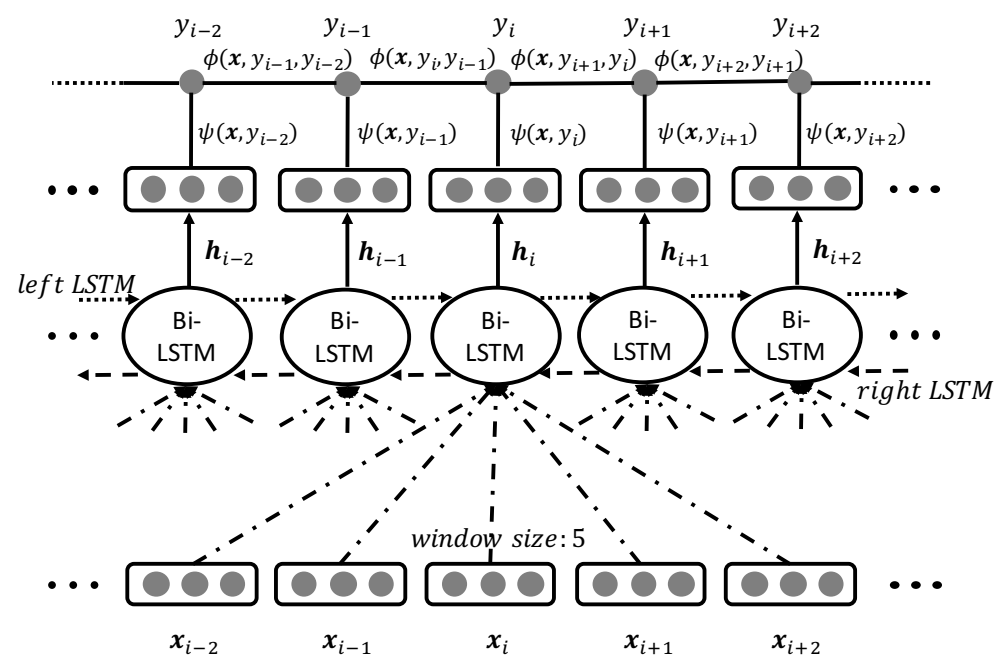

(b) Neural Model Structure

Fig. 1. Model Structures

\section{Method}

The structures of our discrete and neural models are shown in Fig. 1(a) and 1(b) respectively, which are used for all the tasks in this paper. Black and white elements represent binary features for discrete model and gray elements are continuous representation for word/character embedding. The only difference between different tasks are the definition of input and out sequences, and the features used.

The discrete model is a standard CRF model. Given a sequence of input $\boldsymbol{x}=x_{1}, x_{2}, \ldots, x_{n}$, it models the output sequence $\boldsymbol{y}=y_{1}, y_{2}, \ldots, y_{n}$ by calculating two potentials. In particular, the output clique potential shows the correlation between inputs and output labels,

$$
\Psi\left(\boldsymbol{x}, y_{i}\right)=\exp \left(\boldsymbol{\theta}_{\boldsymbol{o}} \cdot \boldsymbol{f}_{\boldsymbol{o}}\left(\boldsymbol{x}, y_{i}\right)\right)
$$

where $\boldsymbol{f}_{\boldsymbol{o}}\left(\boldsymbol{x}, y_{i}\right)$ is a feature vector extracted from $\boldsymbol{x}$ and $y_{i}$, and $\boldsymbol{\theta}_{\boldsymbol{o}}$ is a parameter vector. 

labels,

The edge clique potential shows the correlation between consecutive output

$$
\Phi\left(\boldsymbol{x}, y_{i}, y_{i-1}\right)=\exp \left(\boldsymbol{\theta}_{\boldsymbol{e}} \cdot \boldsymbol{f}_{\boldsymbol{e}}\left(\boldsymbol{x}, y_{i}, y_{i-1}\right)\right)
$$

where $\boldsymbol{f}_{\boldsymbol{e}}\left(\boldsymbol{x}, y_{i}, y_{i-1}\right)$ is a feature vector extracted from $\boldsymbol{x}, y_{i}$ and $y_{i-1}$, and $\boldsymbol{\theta}_{\boldsymbol{e}}$ is a parameter vector.

The final probability of $\boldsymbol{y}$ is estimated as

$$
p(\boldsymbol{y} \mid \boldsymbol{x})=\frac{\prod_{i=1}^{|\boldsymbol{y}|} \Psi\left(\boldsymbol{x}, y_{i}\right) \prod_{j=1}^{|\boldsymbol{y}|} \Phi\left(\boldsymbol{x}, y_{j}, y_{j-1}\right)}{Z(\boldsymbol{x})}
$$

where $Z(\boldsymbol{x})$ is the partition function,

$$
Z(\boldsymbol{x})=\sum_{\boldsymbol{y}} \prod_{i=1}^{|\boldsymbol{y}|} \Psi\left(\boldsymbol{x}, y_{i}\right) \prod_{j=1}^{|\boldsymbol{y}|} \Phi\left(\boldsymbol{x}, y_{j}, y_{j-1}\right)
$$

The overall features $\left\{\boldsymbol{f}_{\boldsymbol{o}}\left(\boldsymbol{x}, y_{i}\right), \boldsymbol{f}_{\boldsymbol{e}}\left(\boldsymbol{x}, y_{i}, y_{i-1}\right)\right\}$ are extracted at each location $i$ according to a set of feature templates for each task.

The neural model takes the neural CRF structure. Compared with the discrete model, it replaces the output clique features $\left.\boldsymbol{f}_{\boldsymbol{o}}\left(\boldsymbol{x}, y_{i}\right)\right)$ with a dense neural feature vector $\boldsymbol{h}_{\boldsymbol{i}}$, which is computed using neural network layer,

$$
\begin{gathered}
\boldsymbol{h}_{\boldsymbol{i}}=\operatorname{BiLSTM}\left(\left(\boldsymbol{e}\left(x_{i-2}\right), \boldsymbol{e}\left(x_{i-1}\right), \boldsymbol{e}\left(x_{i}\right), \boldsymbol{e}\left(x_{i+1}\right), \boldsymbol{e}\left(x_{i+2}\right)\right), \boldsymbol{W}, \boldsymbol{b}, \boldsymbol{h}_{\boldsymbol{i}-\mathbf{1}}\right) \\
\Psi\left(\boldsymbol{x}, y_{i}\right)=\exp \left(\boldsymbol{\theta}_{\boldsymbol{o}} \cdot \boldsymbol{h}_{\boldsymbol{i}}\right)
\end{gathered}
$$

where $\boldsymbol{e}\left(x_{i}\right)$ is the embedding form of $x_{i}$, BiLSTM represents bi-directional LSTM structure for calculating hidden state $\boldsymbol{h}_{\boldsymbol{i}}$ for input $x_{\boldsymbol{i}}$, which considers both the left-to-right and right-to-left information flow in a sequence. The BiLSTM structure receives input of the embeddings from a window of size 5 as shown in Fig. 1(b).

For the neural model, the edge clique is replaced with a single transition weight $\tau\left(y_{i}, y_{i-1}\right)$. The remainder of the model is the same as the discrete model

$$
\begin{aligned}
p(\boldsymbol{y} \mid \boldsymbol{x}) & =\frac{\prod_{i=1}^{|\boldsymbol{y}|} \Psi\left(\boldsymbol{x}, y_{i}\right) \prod_{j=1}^{|\boldsymbol{y}|} \Phi\left(\boldsymbol{x}, y_{j}, y_{j-1}\right)}{Z(\boldsymbol{x})} \\
& =\frac{\prod_{i=1}^{|\boldsymbol{y}|} \exp \left(\boldsymbol{\theta}_{\boldsymbol{o}} \cdot \boldsymbol{h}_{\boldsymbol{i}}\right) \prod_{j=1}^{|\boldsymbol{y}|} \exp \left(\tau\left(y_{j}, y_{j-1}\right)\right)}{Z(\boldsymbol{x})}
\end{aligned}
$$

Here $\boldsymbol{\theta}_{\boldsymbol{o}}$ and $\tau\left(y_{i}, y_{i-1}\right)$ are model parameters, which are different from the discrete model.

The joint model makes a concatenation of the discrete and neural features at the output cliques and edge cliques,

$$
\begin{aligned}
\Psi\left(\boldsymbol{x}, y_{i}\right) & =\exp \left(\boldsymbol{\theta}_{\boldsymbol{o}} \cdot\left(\boldsymbol{h}_{\boldsymbol{i}} \oplus \boldsymbol{f}_{\boldsymbol{o}}\left(\boldsymbol{x}, y_{i}\right)\right)\right) \\
\Phi\left(\boldsymbol{x}, y_{j}, y_{j-1}\right) & =\exp \left(\boldsymbol{\theta}_{\boldsymbol{e}} \cdot\left(\left[\tau\left(y_{j}, y_{j-1}\right)\right] \oplus \boldsymbol{f}_{\boldsymbol{e}}\left(\boldsymbol{x}, y_{j}, y_{j-1}\right)\right)\right)
\end{aligned}
$$


where the $\oplus$ operator is the vector concatenation operation.

The training objective for all the models is to maximize the margin between gold-standard and model prediction scores. Given a set of training examples $\left.\left\{\boldsymbol{x}_{\boldsymbol{n}}, \boldsymbol{y}_{\boldsymbol{n}}\right)\right\}_{n=1}^{N}$, the objective function is defined as follows

$$
L=\frac{1}{N} \sum_{n=1}^{N} \operatorname{loss}\left(\boldsymbol{x}_{\boldsymbol{n}}, \boldsymbol{y}_{\boldsymbol{n}}, \boldsymbol{\Theta}\right)+\frac{\lambda}{2}\|\boldsymbol{\Theta}\|^{2}
$$

Here $\boldsymbol{\Theta}$ is the set of model parameters $\boldsymbol{\theta}_{\boldsymbol{o}}, \boldsymbol{\theta}_{\boldsymbol{e}}, \boldsymbol{W}, \boldsymbol{b}$ and $\tau$, and $\lambda$ is the $L_{2}$ regularization parameter.

The loss function is defined as

$$
\operatorname{loss}\left(\boldsymbol{x}_{\boldsymbol{n}}, \boldsymbol{y}_{\boldsymbol{n}}, \boldsymbol{\Theta}\right)=\max _{\boldsymbol{y}}\left(p\left(\boldsymbol{y} \mid \boldsymbol{x}_{\boldsymbol{n}} ; \boldsymbol{\Theta}\right)+\delta\left(\boldsymbol{y}, \boldsymbol{y}_{\boldsymbol{n}}\right)\right)-p\left(\boldsymbol{y}_{\boldsymbol{n}} \mid \boldsymbol{x}_{\boldsymbol{n}}, \boldsymbol{\Theta}\right)
$$

where $\delta\left(\boldsymbol{y}, \boldsymbol{y}_{\boldsymbol{n}}\right)$ denotes the hamming distance between $\boldsymbol{y}$ and $\boldsymbol{y}_{\boldsymbol{n}}$.

Online Adagrad [59] is used to train the model, with the initial learning rate set to be $\eta$. Since the loss function is not differentiable, a subgradient is used, which is estimated as

$$
\frac{\partial \operatorname{loss}\left(\boldsymbol{x}_{\boldsymbol{n}}, \boldsymbol{y}_{\boldsymbol{n}}, \boldsymbol{\Theta}\right)}{\partial \boldsymbol{\Theta}}=\frac{\partial p\left(\widehat{\boldsymbol{y}} \mid \boldsymbol{x}_{\boldsymbol{n}}, \boldsymbol{\Theta}\right)}{\partial \boldsymbol{\Theta}}-\frac{\partial p\left(\boldsymbol{y}_{\boldsymbol{n}} \mid \boldsymbol{x}_{\boldsymbol{n}}, \boldsymbol{\Theta}\right)}{\partial \boldsymbol{\Theta}}
$$

where $\widehat{\boldsymbol{y}}$ is the predicted label sequence.

Chinese Word Segmentation Features. Table 1 shows the features used in Chinese word segmentation. For "type", each character has five possibilities: 0/Punctuation, 1/Alphabet, 2/Date, 3/Number and 4/others.

Table 1. Feature Templates for Chinese Word Segmentation

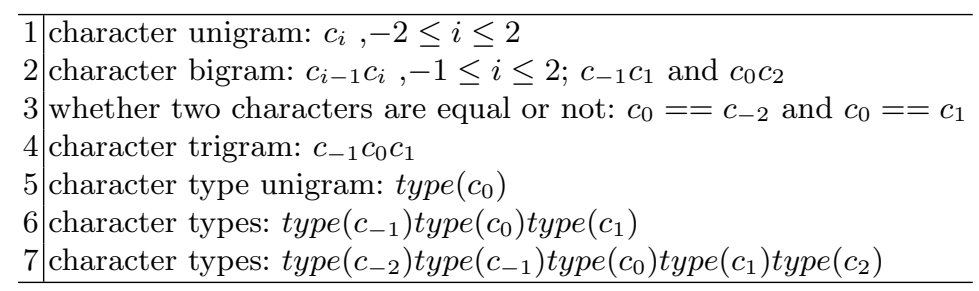

POS Tagging Features. Table 2 lists features for POS tagging task on both English and Chinese datasets. The prefix and suffix include 5 characters for English and 3 characters for Chinese.

NER Features. Table 3 shows the feature template used in English NER task. For "word shape", each character in word is located in one of these four types: number, lower-case English character, upper-case English character and others. "Connect" word has five categories: "of", "and", "for", "-" and other. Table 4 presents the features used in Chinese NER task. We extend the features used in Che et al. 54 by adding part-of-speech information. Both POS tag on 
Table 2. Feature Templates for POS tagging

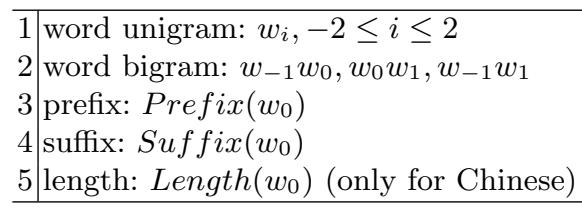

Table 3. Feature Templates for English NER

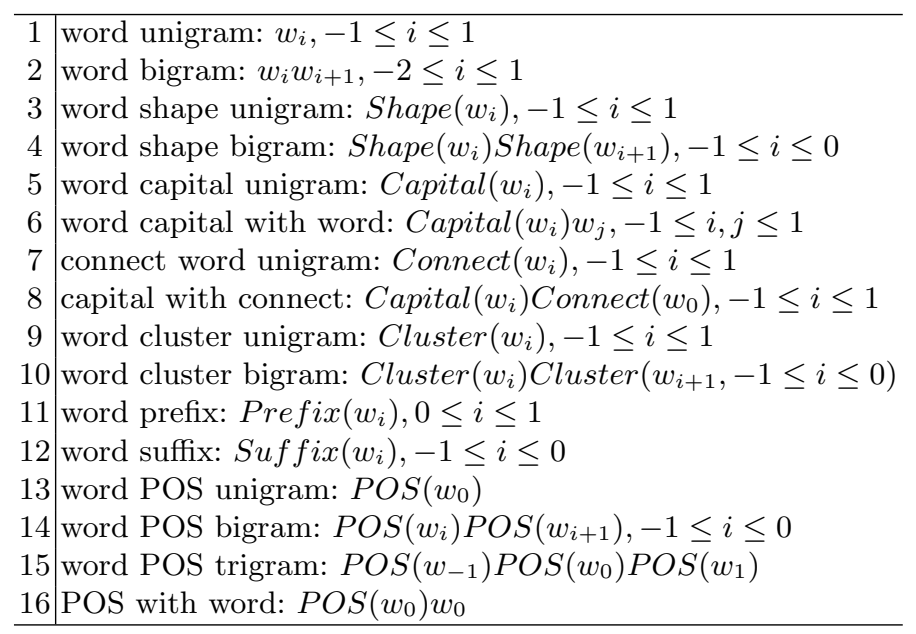

Table 4. Feature Templates for Chinese NER

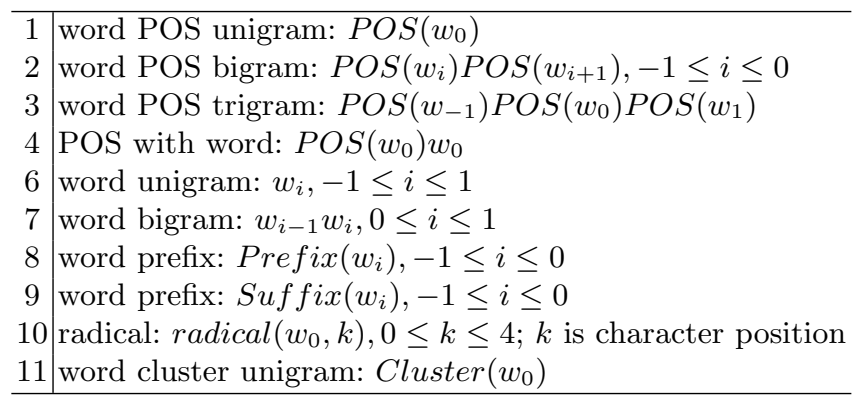

English and Chinese datasets are labeled by ZPar 60, prefix and suffix on two datasets are both including 4 characters. Word clusters in both English and Chinese tasks are same with Che's work [54.

\section{Experiments}

We conduct our experiments on different sequence labeling tasks, including Chinese word segmentation, Part-of-speech tagging and Named entity recognition. 
Table 5. Hyper Parameters

\begin{tabular}{l|c}
\hline Parameter & Value \\
\hline dropout probability & 0.25 \\
wordHiddensize & 100 \\
charHiddensize & 60 \\
charEmbSize & 30 \\
wordEmbSize & 50 \\
wordEmbFineTune & True \\
charEmbFineTune & True \\
initial $\eta$ & 0.01 \\
regularization $\lambda$ & $1 \mathrm{e}-8$ \\
\hline
\end{tabular}

For these three tasks, their input embeddings are different. For Chinese word segmentation, we take both character embeddings and character bigram embeddings for calculating $\boldsymbol{e}\left(x_{i}\right)$. For POS tagging, $\boldsymbol{e}\left(x_{i}\right)$ consists of word embeddings and character embeddings. For NER, we include word embeddings, character embeddings and POS embeddings for $\boldsymbol{e}\left(x_{i}\right)$. Character embeddings, character bigram embeddings and word embeddings are pretrained separately using word2vec 23. English word embedding is chosen as SENNA 25. We make use of Chinese Gigaword Fifth Edition ${ }^{1}$ to pretrain necessary embeddings for Chinese words. The Chinese corpus is segmented by ZPar 60. During training, all these aforementioned embeddings will be fine-tuned. The hyper-parameters in our experiments are shown at Table 5. Dropout 61 technology has been used to suppress over-fitting in the input layer.

\subsection{Chinese Word Segmentation}

For Chinese word segmentation, we choose PKU, MSR and CTB60 as evaluation datasets. The PKU and MSR dataset are obtained from SIGHAN Bakeoff 2005 corpus ${ }^{3}$. We split the PKU and MSR datasets in the same way as Chen et al. [62, and the CTB60 set as Zhang et al. 63. Table 6 shows the statistical results for these three datasets. We evaluate segmentation accuracy by Precision $(\mathrm{P})$, Recall (R) and F-measure (F).

Table 6. Chinese Word Segmentation Datasets Statistics

\begin{tabular}{|c|c|c|c|}
\hline $\begin{array}{c}\text { Segmentation datasets } \\
\text { (sentences) }\end{array}$ & PKU & MSR & CTB60 \\
\hline Train & 17149 & 78226 & 23401 \\
\hline Dev & 1905 & 8692 & 2078 \\
\hline Test & 1944 & 3985 & 2795 \\
\hline
\end{tabular}

\footnotetext{
$\overline{1}$ https://catalog.ldc.upenn.edu/LDC2011T13

${ }^{3}$ http://www.sighan.org/bakeoff2005
} 
The experiment results of Chinese word segmentation is shown in Table 7. Our joint models shown comparable results to the state-of-the-art results reported by Zhang \& Clark [42, where they adopt a word-based perceptron model with carefully designed discrete features. Compared with both discrete and neural models, our joint models can achieve best results among all three datasets. In particular, the joint model can outperform the baseline discrete model by 0.43, 0.42 and 0.37 on PKU, MSR, CTB60 respectively. In order to investigate whether the discrete model and neural model can benefit from each other, we scatter sentence-level segmentation accuracy of two models for three datasets in Fig. 2. As we can see from Fig. 2, some sentences can obtain higher accuracies in the neural model, while other sentences can win out in the discrete model. This common phenomenon among three datasets suggests that the neural model and the discrete model can be combined together to enjoy the merits from each side.

Table 7. Chinese Word Segmentation Results

\begin{tabular}{|c|ccc|cccc|ccc|}
\hline \multirow{2}{*}{ Model } & \multicolumn{3}{|c|}{ PKU } & \multicolumn{3}{c|}{ MSR } & \multicolumn{3}{c|}{ CTB60 } \\
\cline { 2 - 9 } & $\mathrm{P}$ & $\mathrm{R}$ & $\mathrm{F}$ & $\mathrm{P}$ & $\mathrm{R}$ & $\mathrm{F}$ & $\mathrm{P}$ & $\mathrm{R}$ & $\mathrm{F}$ \\
\hline Discrete & 95.42 & 94.56 & 94.99 & 96.94 & 96.61 & 96.78 & 95.43 & 95.16 & 95.29 \\
\hline Neural & 94.29 & 94.56 & 94.42 & 96.79 & $\mathbf{9 7 . 5 4}$ & 97.17 & 94.48 & 95.01 & 94.75 \\
\hline Joint & $\mathbf{9 5 . 7 4}$ & $\mathbf{9 5 . 1 2}$ & $\mathbf{9 5 . 4 2}$ & $\mathbf{9 7 . 0 1}$ & 97.39 & $\mathbf{9 7 . 2 0}$ & $\mathbf{9 5 . 6 8}$ & $\mathbf{9 5 . 6 4}$ & $\mathbf{9 5 . 6 6}$ \\
\hline State-of-the-art & N/A & N/A & 94.50 & N/A & N/A & $\mathbf{9 7 . 2 0}$ & N/A & N/A & 95.05 \\
\hline
\end{tabular}

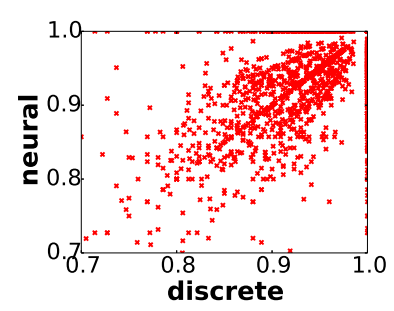

(a) CTB

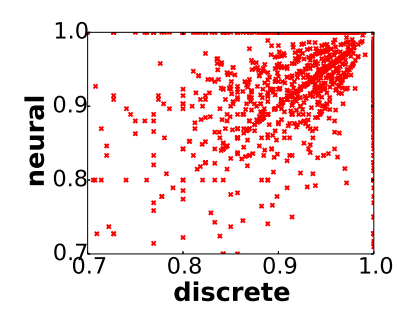

(b) MSR

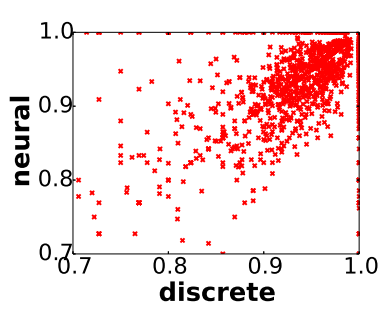

(c) PKU

Fig. 2. Chinese Word Segmentation F-measure Comparisons

\subsection{POS Tagging}

We compare our models on both English and Chinese datasets for the POS tagging task. The English dataset is chosen following Toutanova et al. 64 and Chinese dataset by Li et al. 65. on CTB. Statistical results are shown in Table 8. Toutanova's model [64] exploits bidirectional dependency networks to capture 
both preceding and following tag contexts for English POS tagging task. Li et al. 65] utilize heterogeneous datasets for Chinese POS tagging through bundling two sets of tags and training in enlarged dataset, their system got state-of-the-art accuracy on CTB corpus.

Both the discrete and neural models get comparable accuracies with state-ofthe-art system on English and Chinese datasets. The joint model has significant enhancement compared with separated model, especially in Chinese POS tagging task, with $1 \%$ accuracy increment. Fig. 3 shows the accuracy comparison for the discrete and neural models based on each sentence. There are many sentences that are not located at the diagonal line, which indicates the two models gives different results and have the potential for combination. Our joint model outperforms state-of-the-art accuracy with $0.23 \%$ and $0.97 \%$ on English and Chinese datasets, respectively.

Table 8. POS Tagging Datasets Statistics

\begin{tabular}{|c|c|c|}
\hline $\begin{array}{c}\text { POS tagging datasets } \\
\text { (sentences) }\end{array}$ & English & Chinese \\
\hline Train & 38219 & 16091 \\
\hline Dev & 5527 & 803 \\
\hline Test & 5462 & 1910 \\
\hline
\end{tabular}

Table 9. POS Tagging Results

\begin{tabular}{|c|c|c|}
\hline \multirow{2}{*}{ Model } & English & Chinese \\
\cline { 2 - 3 } & Acc & Acc \\
\hline Discrete & 97.23 & 93.97 \\
\hline Neural & 97.28 & 94.02 \\
\hline Joint & $\mathbf{9 7 . 4 7}$ & $\mathbf{9 5 . 0 7}$ \\
\hline State-of-the-art & 97.24 & 94.10 \\
\hline
\end{tabular}

\subsection{NER}

For the NER task, we split Ontonotes 4.0 following Che et al. 55 to get both English and Chinese datasets. Table 10 shows the sentence numbers of train/develop/test datasets.

We follow Che et al. [54 on choosing both the English and the Chinese datasets. Their work induces bilingual constrains from parallel dataset which gives significant enhancement of F-scores on both English and Chinese datasets.

Our discrete and neural models show comparable recall values compared with Che's results [54 on both datasets. Similar with the previous two tasks, the joint model gives significant enhancement compared with separated models 


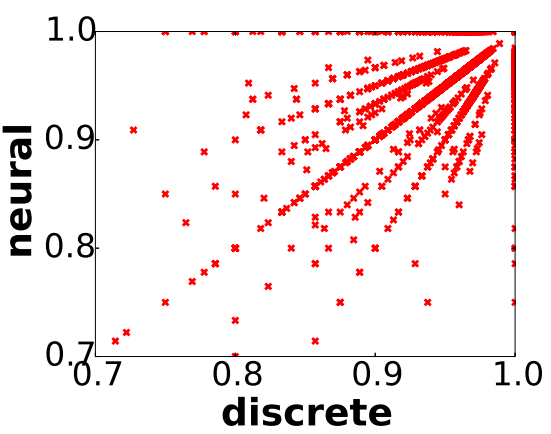

(a) English

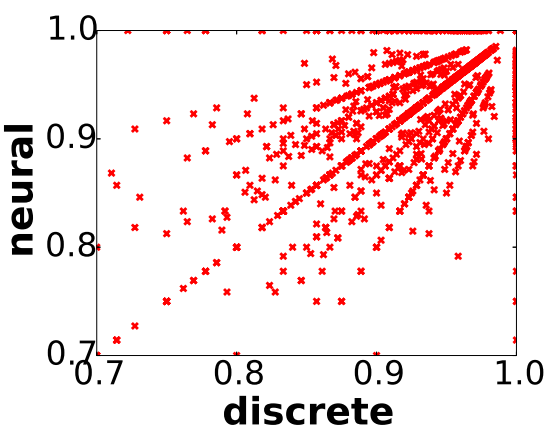

(b) Chinese

Fig. 3. POS Tagging Accuracy Comparisons

Table 10. NER Datasets Statistics

\begin{tabular}{|c|c|c|}
\hline $\begin{array}{c}\text { NER datasets } \\
\text { (sentences) }\end{array}$ & English & Chinese \\
\hline Train & 39262 & 15724 \\
\hline Dev & 6249 & 4301 \\
\hline Test & 6452 & 4346 \\
\hline
\end{tabular}

Table 11. NER Results

\begin{tabular}{|c|ccc|ccc|}
\hline \multirow{2}{*}{ Model } & \multicolumn{3}{|c|}{ English } & \multicolumn{3}{c|}{ Chinese } \\
\cline { 2 - 7 } & $\mathrm{P}$ & $\mathrm{R}$ & $\mathrm{F}$ & $\mathrm{P}$ & $\mathrm{R}$ & $\mathrm{F}$ \\
\hline Discrete & 80.14 & 79.29 & 79.71 & 72.67 & 73.92 & 73.29 \\
\hline Neural & 77.25 & 80.19 & 78.69 & 65.59 & 71.84 & 68.57 \\
\hline Joint & 81.90 & $\mathbf{8 3 . 2 6}$ & $\mathbf{8 2 . 5 7}$ & 72.98 & $\mathbf{8 0 . 1 5}$ & $\mathbf{7 6 . 4 0}$ \\
\hline State-of-the-art & $\mathbf{8 1 . 9 4}$ & 78.35 & 80.10 & $\mathbf{7 7 . 7 1}$ & 72.51 & 75.02 \\
\hline
\end{tabular}

(discrete/neural) on all metrics. This shows that discrete and neural model can identify entities using different indicator features, and they can be complementary with each other. The comparison of sentence F-measures in Fig. 4 confirms this observation.

The joint model outperforms the state-of-the-art on both datasets in the two different languages. The precision of joint models are less than state-of-the-art system. This may be caused by the bilingual constrains in baseline system, which ensures the precision of entity recognition.

\section{Conclusion}

We proposed a joint sequence labeling model that combines neural features and discrete indicator features which can integrate the advantages of carefully designed feature templates over decades and automatically induced features from 


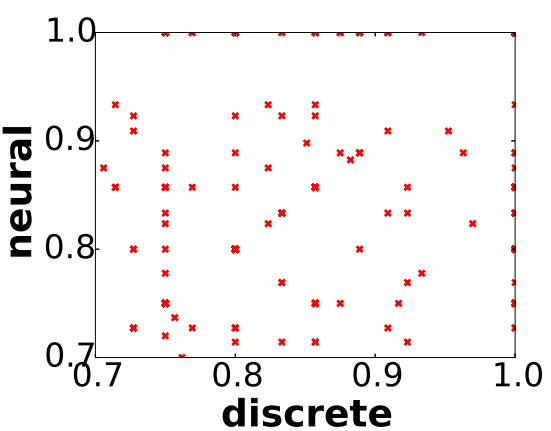

(a) English

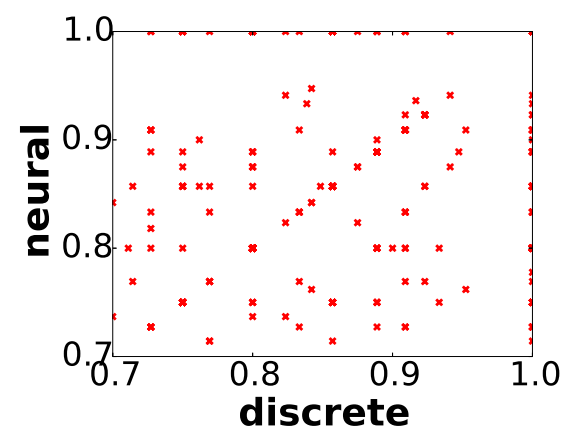

(b) Chinese

Fig. 4. NER F-measure Comparisons

neural networks. Through experiments on various sequence labeling tasks, including Chinese word segmentation, POS tagging and named entity recognition for Chinese and English respectively, we demonstrate that our joint model can unanimously outperform models which only contain discrete features or neural features and state-of-the-art systems on all compared tasks. The accuracy/Fmeasure distribution comparison for discrete and neural model also indicate that discrete and neural model can reveal different related information, this explains why combined model can outperform separate models.

In the future, we will investigate the effect of our joint model on more NLP tasks, such as parsing and machine translation.

\section{Acknowledgments}

We would like to thank the anonymous reviewers for their detailed comments. This work is supported by the Singapore Ministry of Education (MOE) AcRF Tier 2 grant T2MOE201301. 


\section{References}

1. Richard Socher, Cliff C Lin, Chris Manning, and Andrew Y Ng. Parsing natural scenes and natural language with recursive neural networks. In $I C M L$, pages 129 $136,2011$.

2. Danqi Chen and Christopher D Manning. A fast and accurate dependency parser using neural networks. In EMNLP, volume 1, pages 740-750, 2014.

3. David Weiss, Chris Alberti, Michael Collins, and Slav Petrov. Structured training for neural network transition-based parsing. ACL-IJCNLP, pages 323-333, 2015.

4. Chris Dyer, Miguel Ballesteros, Wang Ling, Austin Matthews, and Noah A Smith. Transition-based dependency parsing with stack long short-term memory. $A C L$ IJCNLP, pages 334-343, 2015.

5. Hao Zhou, Yue Zhang, and Jiajun Chen. A neural probabilistic structuredprediction model for transition-based dependency parsing. In $A C L$, pages 1213 $1222,2015$.

6. Greg Durrett and Dan Klein. Neural crf parsing. ACL-IJCNLP, pages 302-312, 2015.

7. Miguel Ballesteros and Xavier Carreras. Transition-based spinal parsing. CoNLL, 2015.

8. Nal Kalchbrenner and Phil Blunsom. Recurrent continuous translation models. In EMNLP, pages 1700-1709, 2013.

9. Kyunghyun Cho, Bart Van Merriënboer, Caglar Gulcehre, Dzmitry Bahdanau, Fethi Bougares, Holger Schwenk, and Yoshua Bengio. Learning phrase representations using rnn encoder-decoder for statistical machine translation. arXiv preprint arXiv:1406.1078, 2014.

10. Ilya Sutskever, Oriol Vinyals, and Quoc VV Le. Sequence to sequence learning with neural networks. In NIPS, pages 3104-3112, 2014.

11. Dzmitry Bahdanau, Kyunghyun Cho, and Yoshua Bengio. Neural machine translation by jointly learning to align and translate. arXiv preprint arXiv:1409.0473, 2015.

12. Wang Ling, Isabel Trancoso, Chris Dyer, and Alan W Black. Character-based neural machine translation. arXiv preprint arXiv:1511.04586, 2015.

13. Sébastien Jean, Kyunghyun Cho, Roland Memisevic, and Yoshua Bengio. On using very large target vocabulary for neural machine translation. $A C L-I J C N L P$, pages $1-10,2015$.

14. Richard Socher, Alex Perelygin, Jean Y Wu, Jason Chuang, Christopher D Manning, Andrew Y Ng, and Christopher Potts. Recursive deep models for semantic compositionality over a sentiment treebank. In $E M N L P$, volume 1631, page 1642, 2013.

15. Duyu Tang, Furu Wei, Nan Yang, Ming Zhou, Ting Liu, and Bing Qin. Learning sentiment-specific word embedding for twitter sentiment classification. In $A C L$, volume 1, pages 1555-1565, 2014.

16. Cicero Nogueira dos Santos and Marra Gatti. Deep convolutional neural networks for sentiment analysis of short texts. In COLING, 2014.

17. Duy-Tin Vo and Yue Zhang. Target-dependent twitter sentiment classification with rich automatic features. In IJCAI, pages 1347-1353, 2015.

18. Meishan Zhang, Yue Zhang, and Duy-Tin Vo. Neural networks for open domain targeted sentiment. In EMNLP, 2015.

19. Richard Socher, Danqi Chen, Christopher D Manning, and Andrew Ng. Reasoning with neural tensor networks for knowledge base completion. In NIPS, pages 926934, 2013. 
20. Mengqiu Wang and Christopher D Manning. Effect of non-linear deep architecture in sequence labeling. In IJCNLP, 2013.

21. Xiao Ding, Yue Zhang, Ting Liu, and Junwen Duan. Deep learning for event-driven stock prediction. In ICJAI, pages 2327-2333, 2015.

22. Granroth-Wilding Mark and Clark Stephen. What happens next? event prediction using a compositional neural network. AAAI, 2016.

23. Tomas Mikolov, Kai Chen, Greg Corrado, and Jeffrey Dean. Efficient estimation of word representations in vector space. arXiv preprint arXiv:1301.3781, 2013.

24. Jeffrey Pennington, Richard Socher, and Christopher D Manning. Glove: Global vectors for word representation. EMNLP, 12:1532-1543, 2014.

25. Ronan Collobert, Jason Weston, Léon Bottou, Michael Karlen, Koray Kavukcuoglu, and Pavel Kuksa. Natural language processing (almost) from scratch. JMLR, 12:2493-2537, 2011.

26. Joseph Turian, Lev Ratinov, and Yoshua Bengio. Word representations: a simple and general method for semi-supervised learning. In $A C L$, pages 384-394, 2010.

27. John Lafferty, Andrew McCallum, and Fernando CN Pereira. Conditional random fields: Probabilistic models for segmenting and labeling sequence data. 2001.

28. Jiang Guo, Wanxiang Che, Haifeng Wang, and Ting Liu. Revisiting embedding features for simple semi-supervised learning. In EMNLP, pages 110-120, 2014.

29. Ji Ma, Yue Zhang, and Jingbo Zhu. Tagging the web: Building a robust web tagger with neural network. In $A C L$, volume 1, pages 144-154, 2014.

30. Mengqiu Wang and Christopher D Manning. Learning a product of experts with elitist lasso. In IJCNLP, 2013.

31. Meishan Zhang and Yue Zhang. Combining discrete and continuous features for deterministic transition-based dependency parsing. EMNLP, pages 1316-1321, 2015.

32. Sepp Hochreiter and Jürgen Schmidhuber. Long short-term memory. Neural computation, 9(8):1735-1780, 1997.

33. Nianwen Xue et al. Chinese word segmentation as character tagging. Computational Linguistics and Chinese Language Processing, 8(1):29-48, 2003.

34. Fuchun Peng, Fangfang Feng, and Andrew McCallum. Chinese segmentation and new word detection using conditional random fields. In Coling, page 562, 2004.

35. Hai Zhao. Character-level dependencies in chinese: usefulness and learning. In EACL, pages 879-887, 2009.

36. Wenbin Jiang, Liang Huang, Qun Liu, and Yajuan Lü. A cascaded linear model for joint chinese word segmentation and part-of-speech tagging. In $A C L, 2008$.

37. Weiwei Sun. A stacked sub-word model for joint chinese word segmentation and part-of-speech tagging. In HLT-ACL, pages 1385-1394, 2011.

38. Yijia Liu, Yue Zhang, Wanxiang Che, Ting Liu, and Fan Wu. Domain adaptation for crf-based chinese word segmentation using free annotations. In EMNLP, pages $864-874,2014$

39. Xiaoqing Zheng, Hanyang Chen, and Tianyu Xu. Deep learning for chinese word segmentation and pos tagging. In EMNLP, pages 647-657, 2013.

40. Wenzhe Pei, Tao Ge, and Chang Baobao. Maxmargin tensor neural network for chinese word segmentation. In $A C L, 2014$.

41. Xinchi Chen, Xipeng Qiu, Chenxi Zhu, and Xuanjing Huang. Gated recursive neural network for chinese word segmentation. In EMNLP, 2015.

42. Yue Zhang and Stephen Clark. Chinese segmentation with a word-based perceptron algorithm. In $A C L$, volume 45, page 840, 2007.

43. Weiwei Sun. Word-based and character-based word segmentation models: Comparison and combination. In Coling, pages 1211-1219, 2010. 
44. Yang Liu and Yue Zhang. Unsupervised domain adaptation for joint segmentation and pos-tagging. In COLING (Posters), pages 745-754, 2012.

45. Adwait Ratnaparkhi et al. A maximum entropy model for part-of-speech tagging. In EMNLP, volume 1, pages 133-142, 1996.

46. Michael Collins. Discriminative training methods for hidden markov models: Theory and experiments with perceptron algorithms. In EMNLP, pages 1-8, 2002.

47. Christopher D Manning. Part-of-speech tagging from $97 \%$ to $100 \%$ : is it time for some linguistics? In Computational Linguistics and Intelligent Text Processing, pages 171-189. Springer, 2011.

48. Cicero D Santos and Bianca Zadrozny. Learning character-level representations for part-of-speech tagging. In ICML, pages 1818-1826, 2014.

49. Juan Antonio Perez-Ortiz and Mikel L Forcada. Part-of-speech tagging with recurrent neural networks. Universitat d'Alacant, Spain, 2001.

50. Zhiheng Huang, Wei Xu, and Kai Yu. Bidirectional lstm-crf models for sequence tagging. 082015.

51. Andrew McCallum and Wei Li. Early results for named entity recognition with conditional random fields, feature induction and web-enhanced lexicons. In $H L T$ NAACL, pages 188-191, 2003.

52. C Hai Leong and N Hwee Tou. Named entity recognition with a maximum entropy approach. In HLT-NAACL, volume 4, pages 160-163, 2003.

53. Vijay Krishnan and Christopher D Manning. An effective two-stage model for exploiting non-local dependencies in named entity recognition. In Coling and $A C L$, pages 1121-1128, 2006.

54. Wanxiang Che, Mengqiu Wang, Christopher D Manning, and Ting Liu. Named entity recognition with bilingual constraints. In $H L T-N A A C L$, pages 52-62, 2013.

55. Lev Ratinov and Dan Roth. Design challenges and misconceptions in named entity recognition. In Coling, pages 147-155, 2009.

56. Cicero dos Santos, Victor Guimaraes, RJ Niterói, and Rio de Janeiro. Boosting named entity recognition with neural character embeddings. In NEWS, 2015.

57. James Hammerton. Named entity recognition with long short-term memory. In Walter Daelemans and Miles Osborne, editors, CoNLL, pages 172-175, 2003.

58. Jason PC Chiu and Eric Nichols. Named entity recognition with bidirectional lstm-cnns. arXiv preprint arXiv:1511.08308, 2015.

59. Yoram Singer John Duchi, Elad Hazan. Adaptive subgradient methods for online learning and stochastic optimization. JMLR, 12:2121-2159, 2011.

60. Yue Zhang and Stephen Clark. Syntactic processing using the generalized perceptron and beam search. Computational linguistics, 37(1):105-151, 2011.

61. Nitish Srivastava, Geoffrey Hinton, Alex Krizhevsky, Ilya Sutskever, and Ruslan Salakhutdinov. Dropout: A simple way to prevent neural networks from overfitting. JMLR, 15(1):1929-1958, 2014.

62. Xinchi Chen, Xipeng Qiu, Chenxi Zhu, Pengfei Liu, and Xuanjing Huang. Long short-term memory neural networks for chinese word segmentation. In EMNLP, pages 1197-1206, Lisbon, Portugal, 2015.

63. Meishan Zhang, Yue Zhang, Wanxiang Che, and Ting Liu. Character-level chinese dependency parsing. ACL, 2014.

64. Kristina Toutanova, Dan Klein, Christopher D Manning, and Yoram Singer. Feature-rich part-of-speech tagging with a cyclic dependency network. In NAACL, pages 173-180, 2003.

65. Li Zhenghua, Chao Jiayuan, Zhang Min, and Chen Wenliang. Coupled sequence labeling on heterogeneous annotations: Pos tagging as a case study. $A C L-I J C N L P$, pages $1783-1792,2015$. 